\title{
INFLUÊNCIA DA ADIÇÃO DE NANOTUBOS DE CARBONO NAS PROPRIEDADES MECÂNICAS DA LIGA AL-2\%NI SOLIDIFICADA UNIDIRECIONALMENTE*
}

\author{
Luiz Gabriel da Silva Nascimento ${ }^{1}$ \\ José Antônio da Silva Souzar ${ }^{2}$ \\ Graziela Cristiane Telles Braga ${ }^{3}$ \\ Fernando Antônio de Sá 4 \\ Marcos Allan Leite do Reis ${ }^{5}$
}

\section{Resumo}

Foi fabricada uma matriz hipoeutética de $\mathrm{Al}-2 \% \mathrm{Ni}$ e adicionado nela concentrações de 0,$5 ; 1,0 ;$ e $1,5 \%$ de nanotubos de carbono de paredes múltiplas (NTCPM). A fabricação do material estudado aconteceu por fundição e resfriamento unidirecional brusco originado grãos predominantemente colunares. Os ensaios mecânicos mostram que concentrações maiores de nanotubo na matriz provocam acréscimo na propriedade mecânica e que em áreas mais distante da base extratora de calor a microdureza aumenta. Na amostra que contém 1,5\% de nanotubos o valor da microdureza em áreas da posição $\mathrm{P} 1$ distante $5 \mathrm{~mm}$ da interface metal/molde é aproximadamente de $120 \mathrm{HV}$, já em áreas da posição $\mathrm{P} 5$ distante $45 \mathrm{~mm}$, se aproxima de $210 \mathrm{HV}$. A resistência a tração é influenciada pela adição de NTC. A matriz possui uma resistência a tração de $64 \mathrm{Mpa}$, enquanto que a adição de 1,5\% de NTC eleva esse valor para $135 \mathrm{MPa}$.

Palavras-chave: Propriedade mecânica; Nanotubos de Carbono de Paredes Múltiplas; Fundição, Solidificação unidirecional.

\section{INFLUENCE OF CARBON NANOTUBES IN MECHANICAL PROPERTIES OF ALLOY AL -2 WITH UNIDIRECTIONAL SOLIDIFICATION}

\section{Abstract}

We made an $\mathrm{Al}-2 \% \mathrm{Ni}$ hypoeutectic matrix with the addition of concentrations of 0.5 , 1.0 and $1.5 \%$ of multi-walled carbon nanotubes (MWCNT). The material under study was manufactured by casting and abrupt unidirectional cooling, generating predominantly columnar grains. The mechanical tests show that higher nanotube content in the matrix leads to an increase in the mechanical property. The tests also reveal an increase in the microhardness in the areas that are more distant from the heat-extracting base. In the sample with $1.5 \%$ of nanotubes, the value of the microhardness in areas of the position P1, $5 \mathrm{~mm}$ away from the metal/mold interface is of approximately $120 \mathrm{HV}$. In areas of the position P5, $45 \mathrm{~mm}$ away, it is about 210 HV. The tensile strength is affected by the addition of CNT. The matrix has a tensile strength of $64 \mathrm{Mpa}$, and the addition of $1.5 \%$ of CNT increases this value to135 MPa. Keywords: Mechanical property; Multi-walled Carbon Nanotubes; Casting, Unidirectional solidification.

1 Engenheiro Mecânico, Doutor em Engenharia de Recursos Naturais, Instituto Federal de Educação, ciência e Tecnologia do estado do Pará - IFPa, Belém - Pará - Brasil.

2 Engenheiro Químico, Doutor em Engenharia de Recursos Naturais, Professor Associado da Universidade Federal do Pará, Belém - Pará-Brasil.

3 Física, Doutora em Física, Técnica de Laboratório, Programa de Pós-Graduação em Física Universidade federal do Pará-UFPa-Belém- Pará- Brasil.

4 Engenheiro Metalúrgico, Mestre em Engenharia Metalúrgica, Doutor, Professor associado II da Universidade Federal do Pará. Professor Universidade federal do Pará, Belém - Pará - Brasil.

5 Físico, Doutor em Engenharia Elétrica, Professor Adjunto II da Universidade Federal do Pará, Campus de Abaetetuba, Abaetetuba - Pará - Brasil. 


\section{INTRODUÇÃO}

A investigação de ligas de alumínio tem um alto grau de importância, principalmente, quando é considerada a intensa procura pelas indústrias automobilística e aeroespacial, por produtos baseados em materiais leves, principalmente ligas de alto desempenho. Um dos aspectos que tornam as ligas de alumínio tão atraentes como materiais de construção mecânica é o fato de o alumínio possuir baixo peso específico e poder combinar-se com a maioria dos metais de engenharia, chamados de elementos de liga. Com essas associações, é possível obter características tecnológicas ajustadas de acordo com a aplicação do produto final. Um dos métodos mais importante para dar forma aos metais é a conformação mecânica, no entanto a técnica de fundição é um dos pedestais da industrialização de um país; sem ela, estabelecida em bases sólidas e de acordo com os recursos regionais, não poderá haver o desenvolvimento racional da produção de utensílios, máquinas e equipamentos. A técnica de solidificação unidirecional permite obter macroestrutura constituída por grãos colunares alinhados numa única direção, Canté, ${ }^{(1)}$ Peres, ${ }^{(2)}$ Sá (3), Silva, ${ }^{(4)}$ Rosa, ${ }^{(5)}$ Rocha, ${ }^{(6)}$.

A nanotecnologia vem despertando muito interesse nas comunidades científicas, e principalmente ao longo das últimas décadas muitos esforços foram feitos no sentido de atingir o tão desejado controle em nível atômico e molecular sobre os processos industriais. Com o surgimento dos materiais nanométricos, e em função deles, novas técnicas de caracterização foram projetadas e implementadas Kuzmany, ${ }^{(7)}$ Dos Reis, ${ }^{(8)}$. Existem varias mecanismos de fabricação de nanotubos de carbono, Aguilar, ${ }^{(9)}$ Barreiro, ${ }^{(10)}$ Cheng, ${ }^{(11)}$ lijima, ${ }^{(12)}$; Oberlin, ${ }^{(13)}$, Schnitzler, ${ }^{(14)}$ e segundo Herbst, ${ }^{(15)}$ os nanotubos implicam em grande potencial de aplicações.

A necessidade de controlar as morfologias apropriadas para sua utilização fazem da pesquisa nesta área do conhecimento um trabalho de característica eminentemente multidisciplinar, envolvendo fatores que definem o sucesso de suas aplicações, tais como rota de síntese, processamento em formas variadas e qualidade dos NTC. Desde sua descoberta, os nanotubos tem despertado grande interesse em pesquisas, o que aumentou exponencialmente o número de publicações científicas anuais. Atualmente, estudos sobre os nanotubos atravessam as fronteiras da Física, da Química, da Ciência de Materiais com varias aplicaçe até mesmo da Biologia, desenvolvendo-se em ampla diversidade de aplicações; para tanto, o conhecimento da constituição dos nanotubos e o domínio dos processos de funcionalização faz-se primordial, Loos, ${ }^{(16)}$, Andrews, ${ }^{(17)}$. O objetivo deste estudo, motivado pelas varias aplicações e resultados relevantes da aplicação dos nanotubos de carbono em outros materiais é de verificar a influencia de concentrações varaidas destes materiais nas propriedades mecânicas da matriz hipoeutéica $\mathrm{Al}-2 \% \mathrm{Ni}$ solidificadas unidirecionalmente

\section{MATERIAIS E MÉTODOS}

\subsection{Descrição do dispositivo de solidificação}

Foi projetado um dispositivo de solidificação unidirecional com intuito de após a solidificação os grãos apresentem direções orientadas. O sistema de aquecimento do dispositivo e compostos por quatro resistência de $400 \mathrm{~W}$ a $220 \mathrm{~V}$. A etapa de aquecimento exige aproximadamente 1 (uma) hora para se atingir a temperatura de $700^{\circ} \mathrm{C}$. Desta forma, o dispositivo atinge uma temperatura interna em sua câmara o suficiente para manter no estado liquido as ligas metálicas que se situa dentro da 
lingoteira de fusão. O Reservatório possui capacidade $100 \mathrm{~L}$ de água que provoca a solidificação forçada do material. O mesmo é suspenso por um suporte feito de barras de ferro com uma altura de 1,80 m.

A água circula até a interface metal/molde pela ação da gravidade, através da mangueira de ligação do reservatório ao dispositiv. Depois do contato, a principio em forma de vapor e depois líquido, segue para outra região onde será descartada para o ambiente. A vazão máxima do sistema de resfriamento é em média de $14 \mathrm{l} / \mathrm{min}$, estando o reservatório completamente preenchido pela água, mas é possível variar a velocidade do líquido, por isso o sistema pode ter taxa de resfriamento controlada. A Figura 1 mostra o desenho em três dimensões do forno, podendo-se visualizar as resistências, o sistema de refrigeração com as tubulações de entrada (superior) e saída (inferior) de água e a tampa bipartida, responsável pela isolação térmica superior e passagem dos termopares para medição de temperatura da liga já fundida. As variáveis do dispositivo, principalmente pressão e vazão do líquido de arrefecimento estão descritas na Tabela 1, pois elas influenciam diretamente na qualidade da propriedade mecânica dos materiais solidificados.

Tabela 1. Variáveis do sistema de solidificação unidirecional

\begin{tabular}{ll}
\hline Vazão de H20 & $\mathbf{1 4 ~ I / m i n ~}$ \\
\hline Vazão de Argônio & $6 \mathrm{I} / \mathrm{min}$ \\
Pressão na base da lingoteira & $17,658 \mathrm{kPa}$ \\
Potência total do sistema de aquecimento & $1600 \mathrm{~W}$ \\
Tensão & $220 \mathrm{~V}$ \\
\hline
\end{tabular}

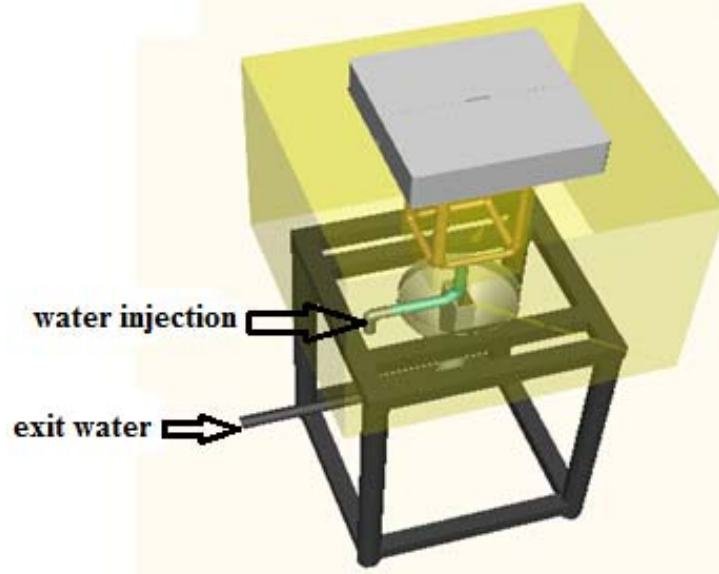

Figura 1. Termopares acoplados na material solidificado para retirada de dados térmicos.

\subsection{Processo de fabricação de fabricação dos lingotes com NTC}

Lingotes de matriz Al-2\%Ni com furos não passantes que continham NTC, o foram levados dentro de um cadinho revestido com alumina a um forno mufla a uma temperatura de $750^{\circ} \mathrm{C}$ até sua completa fusão. A alumina foi usada como revestimento para proteger o material, da possível entrada de contaminantes na matriz. A homogeneização do material foi feita por uma haste oca de cobre revestida de alumina, para não existir o contado entre o cobre e alumínio. Por essa haste passava argônio objetivando facilitar a mistura e evitar defeitos no material, causados por gases da atmosfera. O processo aconteceu com uma vazão de 14 I/min de argônio. Todo o processo era feito em menos de 10 segundos, com intuito 
de não deixar o nanotubo perder massa. Rapidamente o cadinho voltava ao forno e depois da segunda agitação a massa de alumínio líquido com níquel mais nanotubo de carbono era direcionada até a lingoteira, que se encontrava dentro do dispositivo de solidificação. Posteriormente a todo esse processo, a câmara de aquecimento do dispositivo era fechada, dai em diante o sistema de refrigeração era acionado pela válvula de controle de fluxo de água e acontecia a solidificação unidirecional, a temperatura de acionamento do sistema hidráulico era de aproximadamente $700{ }^{\circ} \mathrm{C}$

\subsection{Procedimentos para ensaios Metalográficos e Mecânicos}

As superfícies dos materiais processados foram polidas e secadas para que a água do processo de polimento não interferisse na qualidade do reagente $e$ comprometesse o ataque químico. As peças foram atacadas quimicamente, por passagem do reagente na superfície do material com uma solução aquosa de $5 \mathrm{ml}$ $\mathrm{HF}, 30 \mathrm{ml} \mathrm{HNO} 3,60 \mathrm{ml} \mathrm{HCl}$ e $5 \mathrm{ml} \mathrm{H} 2 \mathrm{O}$ (Poulton) até a completa revelação da macroestrutura. $\mathrm{O}$ ataque químico foi executado por um tempo de aproximadamente dez segundos, e quando revelada a estrutura de solidificação a peça foi lavada a água corrente e submetida novamente ao processo de secagem.

Com o objetivo de caracterizar mecanicamente os materiais, fabricados nesse trabalho, em especifico o limite de resistência a tração, foram fabricados vinte e cinco pequenos corpos de prova sendo cinco para cada material: Al comercialmente puro, Al- $\% 2 \% \mathrm{Ni}$; Al-2\% Ni 0,5\% NTC; Al-2\% Ni 1,0\% NTC e Al-2\% Ni 1,5\% NTC. Os lingotes foram seccionados longitudinalmente e fabricados os corpos de prova tomando a forma geométrica do modelo da Figura. Foi usada para o ensaio mecânico de tração uma máquina universal marca modelo WDW-10EIII 1000 kgf, a velocidade de ensaio foi $0,2 \mathrm{~mm} / \mathrm{s}$, os dados captados por um software e os resultados revelados para a confecção dos gráficos e análise das propriedades.

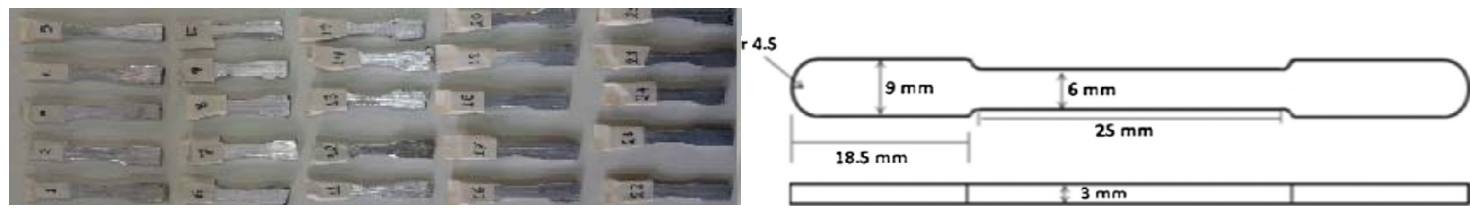

Figura 2. Dimensões e geometria dos corpos de prova usados no ensaio de tração.

Para realizar as medidas de microdureza foi utilizado um microdurômetro marca extração de calor. Foi utilizado um microscópio eletrônico de varredura (VEGA3, TESCAN) para verificar a disposição dos nanotubos na matriz. As análises foram executadas na posição P5 do nanocompósito Al-2\%Ni Al-2\%Ni 1,5\% NTC. Foi feito um mapeamento de carbono com detector EDS swift ED3000 x-ray para análise semi-quantitativas sobre as áreas de P1, e P5 com intuito de verificar se a concentração de carbono e a mesma ao longo do lingote.

Descrever brevemente os equipamentos e os procedimentos utilizados, assim como a literatura e os métodos estatísticos empregados, quando for o caso.

\section{RESULTADOS E DISCUSSÃO}

\subsection{Macra e Micro estrutura após Solidificação Unidirecional}

A Figura 3 mostra as macrografias dos materiais estudados, após ataque químico realizado, onde se pode visualizar a unidirecionalidade do fluxo de calor em relação à base do molde onde concordam com trabalhos encontrados na literatura, Canté, ${ }^{(1)}$ 
Peres, ${ }^{(2)}$. A morfologia das macroestruturas observada para as ligas utilizadas neste trabalho foi predominantemente colunar e vertical, comprovando assim a direcionalidade e eficiência em que o processo de solidificação unidirecional aconteceu, os grãos tem a direção do fluxo transiente de calor. A liga $\mathrm{Al}-2 \% \mathrm{Ni}$ apresenta grande contraste quando comparada sua macroestrutura ao nanocompósito Al-2\%Ni 1,5\% NTC, uma vez que este ultimo apresenta grãos mais refinados. Quanto maior a concentração de NTC nos materiais mais refinados são os grãos.

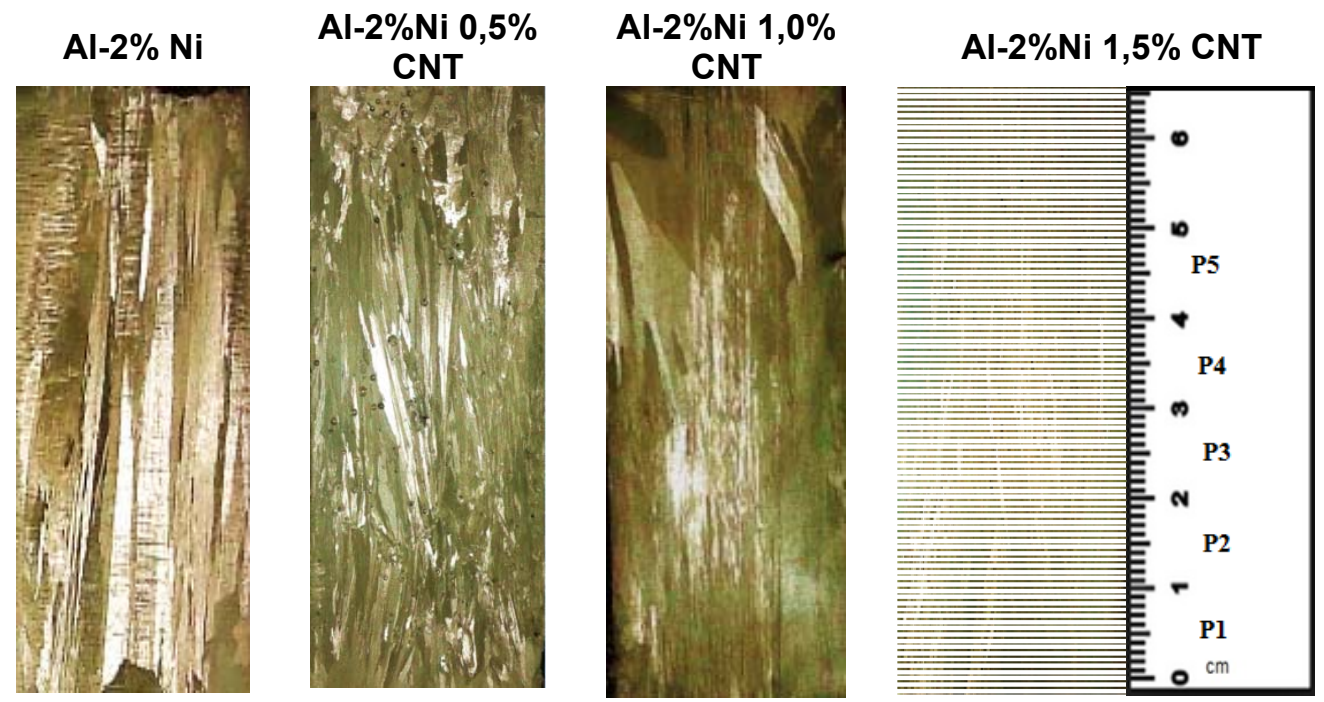

Figura 3. Imagem da macroestrutura revelada após ataque químico dos materiais de estudo, que revela a unidirecionalidade dos grãos influencia da solidificação.

Foi possível capturar imagens que mostram a forma de como os nanotubos estão comportados na matriz. A Figura 4 (a), (b) e (c), são o local e a aproximação das imagens que mostram as nonoestruturas é possível observar o aglomerado de nanotubos na matriz que atua como reforço.

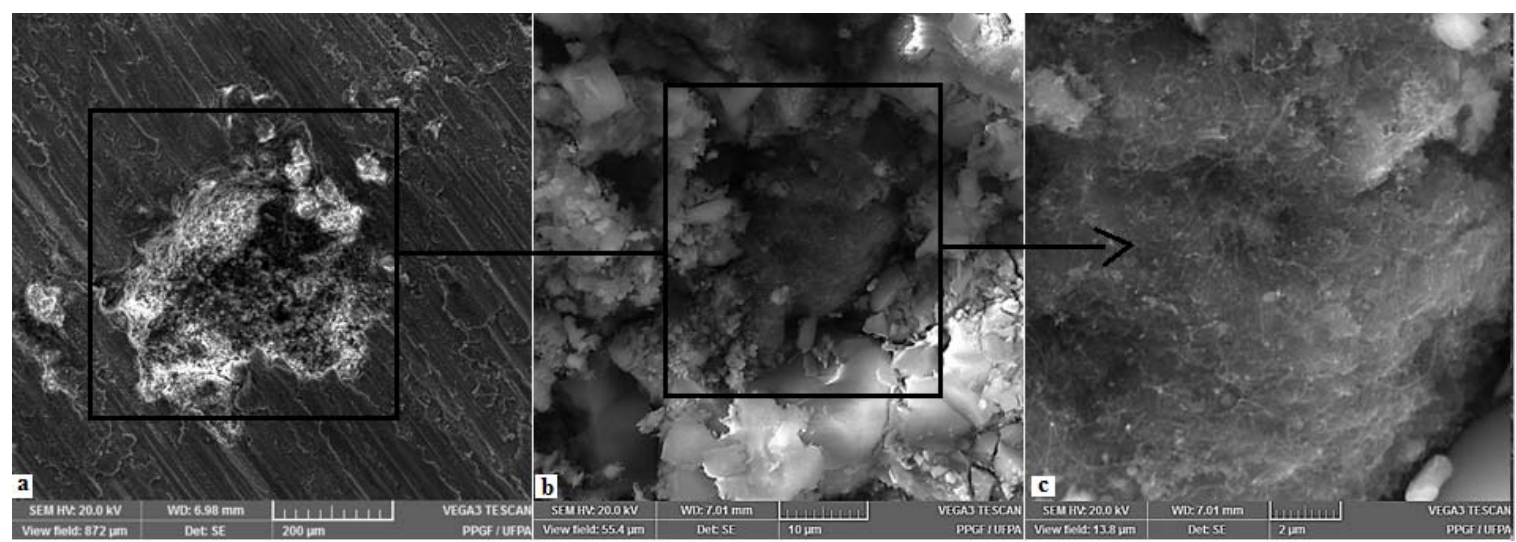

Figura 4. (a), (b): Local e aproximação de analise de MEV e nanotubos de carbono na matriz de Al$\mathrm{Ni}$.

Na figura 5, encontramos o mapeamento da distribuição de carbono na matriz de $\mathrm{Al}$ $2 \%$ Ni 1\% NTC. A Figura 5 (b) e (d), indica que existe contraste entre a distribuição de carbono na área da posição $\mathrm{P} 1$ em relação à área posição $\mathrm{P} 5$, observa-se maior concentrações de carbono para a região superior do lingote. As Figuras 5 (a) e (c), são mapeamentos dos elementos níquel e carbono espalhados na matriz de estrutura dendrítica. 


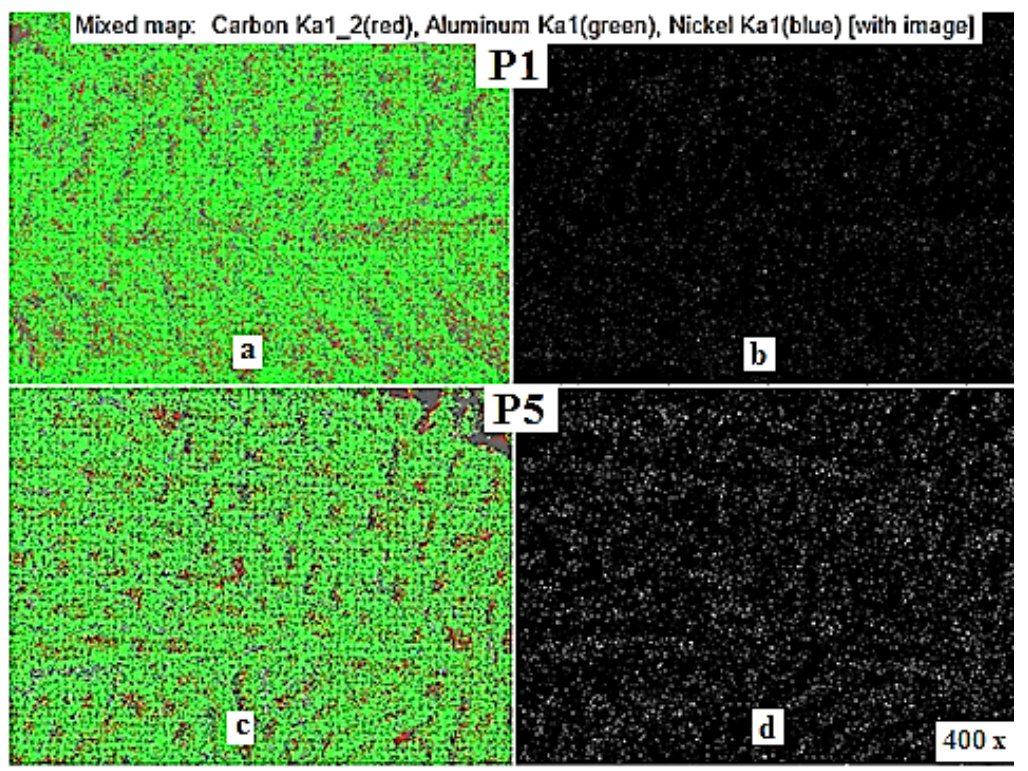

Figura 5 (a), (b), (c) e (d): Contraste da quantidade de Carbono entre área da posição P1 e área da posição P5 no nonocrocompósito Al- 2\%Ni 1\% CTN (400x).

\subsection{Propriedades Mecânicas}

Os gráficos da Figura 7 mostram, que para cada material estudado, há evolução da microdureza quando o teste mecânico é aplicado em áreas que vão se distanciado da interface extratora de calor, ou seja, a resistência aumenta em regiões mais afastadas da interface aonde ocorreu a transferência de calor. Esse comportamento é explicado pelo fato de que durante o processo de resfriamento brusco e solidificação os NTC estão se afastando para as regiões mais distantes da base de extração de calor, explicado pelo mapeamento de carbono onde mostra maior concentração de carbono em regiões superiores do lingote. Se o soluto, apresentar uma densidade significativamente diferente ele pode flutuar por cima do restante do material fundido, influenciado pelo fenômeno convectivo comum nesse processo de solidificação. Tal comportamento dos nanotubos é bastante interessante, pois possibilita criar projetos de ligas de alumínio, com injeção de nanotubos por fusão e solidificação com superfícies mais resistentes, sujeitas aos mais elevados esforços mecânicos em indústria aeronáutica, militar, máquinas e equipamentos. O gráfico da Figura 6 mostra que quanto maior a concentração de NTC na matriz maior será a resistência mecânica do material e em que em pontos mais afastados da base do lingote a propriedade mecânica também aumenta. Na amostra que contém $1,5 \%$ de nanotubos o valor de microdureza na posição P1 $(5 \mathrm{~mm})$ é aproximadamente de 120 $\mathrm{HV}$, já na posição P5 (45 mm) chega quase a $210 \mathrm{HV}$. O valor da microdureza também aumenta com a concentração de NTC na matriz. O valor de Microdureza da Matriz na posição P5 chega próximo a $105 \mathrm{HV}$, pra essa mês'ma posição na matriz com a inserção de $1,5 \%$ de NTC esse valor quase chega a dobrar, sendo de aproximadamente $200 \mathrm{HV}$. 


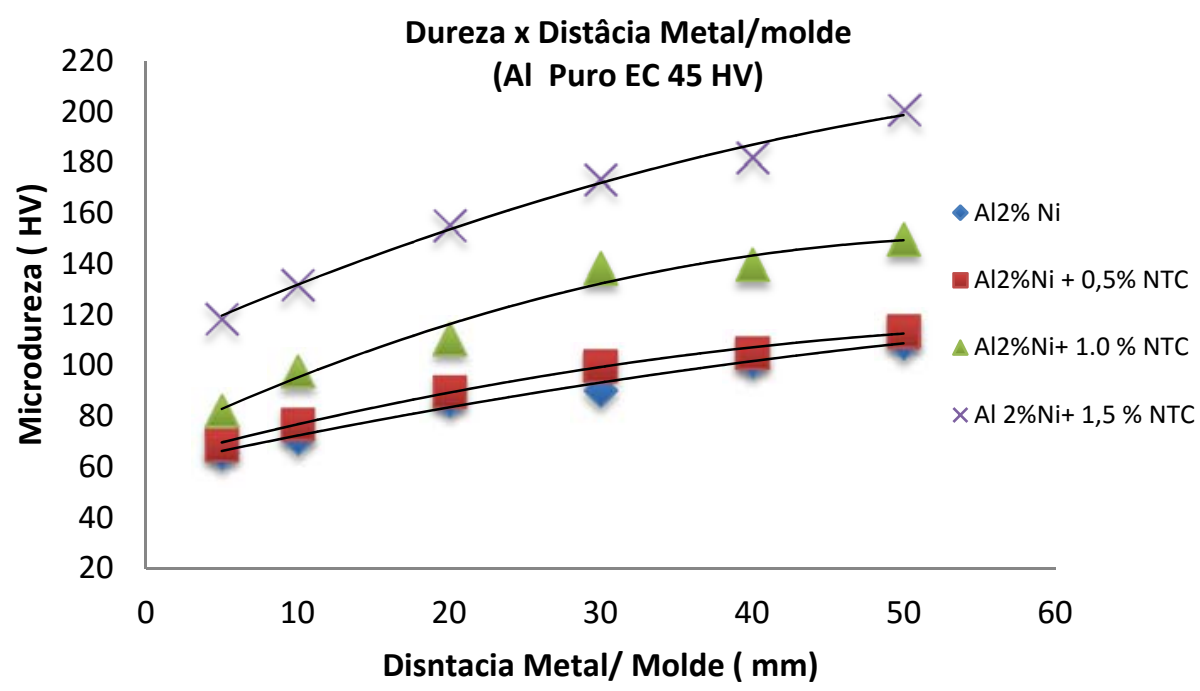

Figura 6. Evolução da microdureza relacionado à concentração de NTC na matriz metálica e a distância da base extratora de calor.

A Figura 7 deixa claro o aumento da resistência a tração quando é adicionado na matriz concentrações diferenciadas de nanotubo de carbono. Conforme aumenta a concentração de NTC na liga AINi há aumento na resistência do material. A matriz possui uma resistência a tração próxima de $64 \mathrm{Mpa}$ enquanto que a adição de 1,5\% de NTC eleva esse valor para perto de $135 \mathrm{MPa}$. Isso acontece porque os nanotubos de carbono por possuem alta resistência mecânica, Herbst, ${ }^{(15)}$ e divide a carga aplicada com a matriz proporcionando um material mais resistente. Os resultados que norteiam esse trabalho estão de acordo com os resultados já encontrados na literatura, uma vez que os nanotubos melhoram a propriedade mecânica da matriz, Schnitzle, ${ }^{(14)}$ Loos, ${ }^{(16)}$.

\section{Resitência em relação a concentração de nanotubo}

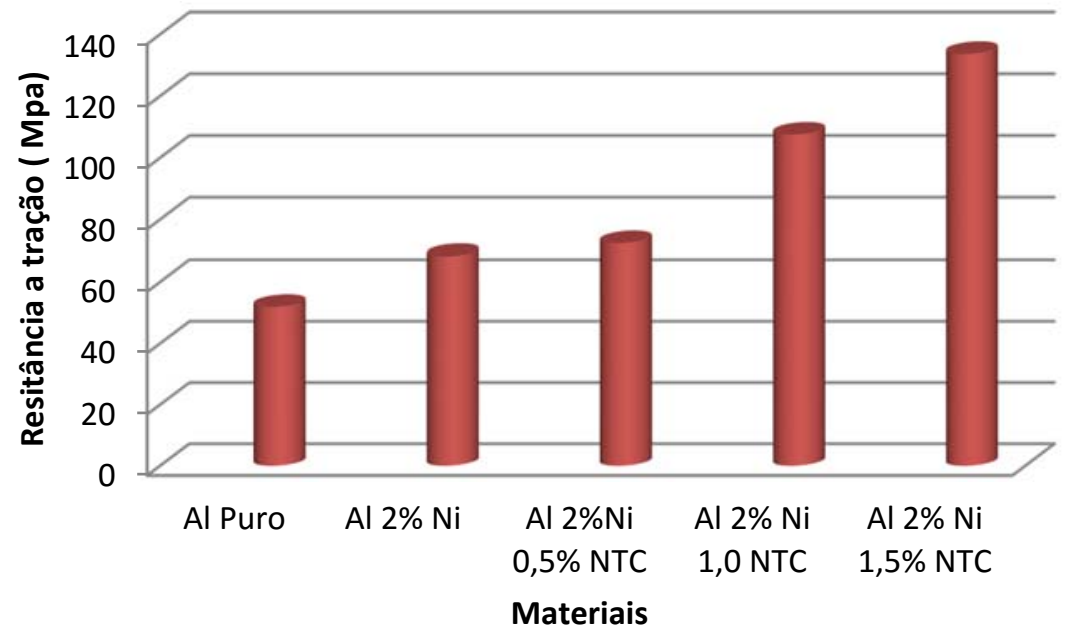

Figura 7. Comportamento da Resistência Mecânica em relação ao aumento da concentração de NTC 


\section{CONCLUSÃO}

As macroestruturas de solidificação dos materiais estudados vem apresentar grãos predominantemente colunares, onde nota-se grãos mais refinados no material possuem maior concentração de nanotubos.

A microdureza nas regiões mais distante da base extratora de calor do lingote tem valores maiores que na base, isso devido a difusão dos nanotubos para essas regiões mais afastadas. Esse comportamento dos nanotubos é muito interessante, pois possibilita criar projetos de ligas de alumínio por fusão e solidificação com superfícies mais resistentes aos mais elevados esforços mecânicos em indústria aeronáutica, militar, máquinas e equipamentos.

$\mathrm{Na}$ amostra que contém $1,5 \%$ de nanotubos o valor de microdureza na posição P1 $(5 \mathrm{~mm})$ é aproximadamente de $120 \mathrm{HV}$, já na posição P5 $(45 \mathrm{~mm})$ chega quase a $210 \mathrm{HV}$. O valor da microdureza também aumenta com a concentração de NTC na matriz. O valor de Microdureza da Matriz na posição P5 chega próximo a $105 \mathrm{HV}$, pra essa mês'ma posição na matriz com a inserção de $1,5 \%$ de NTC esse valor quase chega a dobrar, sendo de aproximadamente $200 \mathrm{HV}$.

A resistência a tração é influenciada pela adição de NTC, pois conforme aumenta a concentração de NTC na liga AINi, também aumenta a resistência do material. A matriz possui uma resistência a tração próxima de $64 \mathrm{Mpa}$, enquanto que a adição de $1,5 \%$ de NTC eleva esse valor para aproximadamente $135 \mathrm{MPa}$. Isso acontece porque os nanotubos de carbono por possuem alta resistência mecânica divide a carga aplicada com a matriz, proporcionando um material mais resistente.

Descrição das principais conclusões.

\section{Agradecimentos}

Em especial ao Instituto Federal do Pará (IFPa), ao Programa de Pós- Graduação em Engenharia de Recursos Naturais de Amazônia- Laboratório de Microscopia Eletrônica-PRODERNA-UFPA e ao Laboratório de Física / Pós-Graduação/ UFPA.

\section{REFERÊNCIAS}

1 CANTÉ, M. V. Solidificação transitória, microestrutura e propriedades de ligas Al-Ni. Campinas: Faculdade de Engenharia Mecânica, Universidade Estadual de Campinas, 2009. (Tese de Doutorado).

2 PERES, M. D., SIQUEIRA, C. A., GARCIA, A. Macrostructural and microstructural development in $\mathrm{Al}-\mathrm{Si}$ alloys directionally solidified under unsteady-state conditions. Journal of Alloys and Compounds, v. 381, p. 168-181, 2004.

3 Sá. F, Rocha O. F. L.; Siqueira C. A.; Garcia A. The effect of solidification variables o tertiary dendrite arm spacing in unsteady-state directional solidification of $\mathrm{Sn}-\mathrm{Pb}$ andbAl-Cu alloys. Materials Science and Engineering A, v. 373, pp. 131-138, 2004

4 SILVA, J.N.S. Projeto, Construção e Aferição de um Dispositivo de Solidificação Unidirecional Horizontal Refrigerado à Água. Belém: Departamento de Engenharia Mecânica, Universidade Federal do Pará, 2007. (Dissertação de mestrado).

5 Rosa, D. M. Estruturas celulares, transição celular/dendrítica e estruturas dendríticas na solidificação unidirecional transitória. Campinas: Faculdade de Engenharia Mecânica, Universidade Estadual de Campinas, 2007. (Tese de Doutorado).

6 ROCHA, O. L, GOMES, L.G, MOUTINHO, D.J, FERREIRA, I.L,GARCIA,A. The columnar to equiaxed transition in the directional solidification of aluminum based multicomponent alloys. REM - Revista Escola de Minas, 2015, vol.68, n.1, pp. 85-90. 
$7 \quad$ KUZMANY, H.; KUKOVECZ, A.; SIMON, F.; HOLZWEBER, M.; KRAMBERGER, C.; PICHLER,T. Functionalization of carbon nanotubes. Synthetic Metals, v. 141, p. 113122, 2004.

8 Dos Reis, Marcos A. L. ; Saraiva, A. C. F. ; Vieira, M. F. G. ; Del Nero, Jordan . Study of Ink Paper Sensor Based on Aluminum/Carbon Nanotubes Agglomerated Nanocomposites. Journal of Nanoscience and Nanotechnology , v. 12, p. 6955-6960, 2012

9 AGUILAR-ELGUÉZABAL, A.; ANTÚNES, W.; ALONSO, G.; PARAGUAY DELGADO, F.; ESPINOSA, F.; MIKI-YOSHIDA, M. Study of carbono nanotubes synthesis by spray pyrolysis and model of growth. Diamond and Related Materials, v. 15, p. 1329-1335, 2006

10 BARREIRO, A.; HAMPEL, S.; RUMMELI, M. H.; KRAMBERGER, C.; GRUNEIS, A.; BIEDERMANN, K.; LEONHARDT, A.; GEMMING, T.; BUCHNER, B.; BACHTOLD, A.; PICHLER, T. Decomposition of Ferrocene as a Method for Production of Single-Walled Carbon Nanotubes without Additional Carbon Sources, Journal of Physical Chemistry B, v. 110, p. 20973-20977, 2006.

11 CHENG, H. M.; SUN, X.; LI, F.; BROWN, S. D. M.; PIMENTA, M. A.; MARUCCI, A.; DRESSELHAUS, G.; DRESSELHAUS, M. S. Bulk morphology and diameter distribution of single-walled carbon nanotubes synthesized by catalytic decomposition of hydrocarbons. Chemical Physics Letters, v. 289, p. 602, 1998.

1212 IIJIMA, S.; Helical microtubules of graphitic carbon. Nature, v. 354, p. 56- 58, 1991.

1313 OBERLIN, A.; ENDO, M.; KOYAMA, T. Filamentous growth of carbon through benzene decomposition. Journal of Crystal Growth, v. 32, p. 335-349, 1976.

14 SCHNITZLER, M.C. Síntese e Caracterização de Nanotubos de Carbono Utilizando Precursosres Organometálicos. Programa de Pós Graduação em Química, Universidade Federal do Paraná, 2007. (Tese de Doutorado).

15 Herbst, M. H.; Macêdo, M. I. F; Rocco, A.M. Tecnologia dos Nanotubos de Carbono: Tendência e Perspectiva de uma area multidisciplinar. Quim. Nova, Vol. 27, No. 6, 986992, Rio de Janeiro 2004.

1616 LOOS, R; COELHO, L. A. F; PEZZIN, S. H ; AMICO, S. C. Effect of carbon nanotubes addition on the mechanical and thermal properties of epoxy matrices. Mat. Res., 2008, vol.11, n.3, pp. 347-352.

17 ANDREWS, R.; JACQUES, D.; RAO, A. M.; DERBYSHIRE, F.; QIAN, D.; FAN, X.; DICKEY, E. C.; Continuous production of aligned carbon nanotubes: a step closer to commercial realization, Chemical Physics Letters, v.303, p. 467, 1999. 